\title{
Validation of Geant4 Physics Models Relevant for Space Radiation
}

\author{
V. Satya Prakash \\ Department of Physics, Government Degree College, Medak, India
}

Email address:

satyaprakashvpet@yahoo.co.in

To cite this article:

V. Satya Prakash. Validation of Geant4 Physics Models Relevant for Space Radiation. American Journal of Environmental Protection. Vol. 6, No. 1, 2017, pp. 18-25. doi: 10.11648/j.ajep.20170601.13

Received: January 20, 2017; Accepted: February 15, 2017; Published: March 6, 2017

\begin{abstract}
In order to use GEANT4 toolkit in the energies relevant to the space radiation it has been tested few of electromagnetic and hadronic models of it by comparing simulated values with NIST data and other experimental data available. For the validation of electromagnetic models energy-loss and electronic stopping powers are considered whereas for the validation of hadronic models, isotope production cross-sections and total fragmentation cross-sections are considered. The stopping power values simulated for protons in Al are agreeing very well with NIST database values. The energy-loss and residual energy values simulated for alpha particles in $\mathrm{Si}$ and $\mathrm{Al}$ respectively are in good agreement with experimental values at high energies and low-thicknesses of target materials. The stopping power values of alpha particles and $\mathrm{Fe}$ ions in $\mathrm{Al}$ are also agreeing well with tabulated values at the small thickness of target materials. The proton - proton production cross-section values for liquid hydrogen and polyethylene are within the limits of experimental errors. Although total fragmentation crosssections for $\mathrm{Fe}$ ions in polyethylene and aluminum are not agreeing with the experimental values at low energies, they are agreeing at the peak of GCR spectrum which is around $1 \mathrm{GeV} /$ nucleon. So the selected physics models used in the present simulation work can be used for the space radiation protection studies.
\end{abstract}

Keywords: Geant4, Space Radiation Protection, Electromagnetic Interactions, Hadronic Interactions

\section{Introduction}

Geant4 - Geometry And Tracking (version 4) is a toolkit for the simulation of particle interactions and transport through the matter [1]. It is modular software written in object oriented frame work. It is capable of dealing with the interactions of leptons, photons, hadrons and ions. It provides a number of physics models to deal with the particle and matter interactions in the wide range of energies corresponding from thermal neutrons to cosmic rays $[2,3,4$, $9,12]$. So it is used in diversified areas like medical physics and space radiation protection etc. apart from detector simulations.

Space radiation is made of Galactic Cosmic Rays (GCR), Solar Particle Events (SPE) and Tapped radiation. The GCR radiation originating from outside the solar system consists of $98 \%$ baryons and $2 \%$ electrons. The baryonic component in turn consists of $85 \%$ protons, $14 \%$ helium nuclei and $1 \%$ heavier nuclei with typical energies up to $10^{20} \mathrm{eV}$ [7]. However, the number of such high energy particles near the earth orbit is very small but the typical energies of high intensity particles extend from $100 \mathrm{MeV}$ to $10 \mathrm{GeV}$ [11]. The peak of the GCR spectra is around the $1 \mathrm{GeV} /$ nucleon for all ions [7]. The SPE radiation originating from Coronal Mass Ejections of the Sun consists of the majority of protons and a small fraction of heavier nuclei with energies up to several $\mathrm{GeV}$. The trapped radiation originating from the interactions of GCR and SPE radiation with earth's geomagnetic field appear in the form of van Allen Belts around the earth. Inner belt of van Allen Belts consists of protons and electrons, whereas the outer belt consists of only electrons. The typical energies of electrons are up to $7 \mathrm{MeV}$ whereas the energies of protons are up to $600 \mathrm{MeV}$ [7].

To do any meaningful analysis of space radiation protection it is necessary to validate the chosen Geant4 physics models in the energy range of $100 \mathrm{MeV}-10 \mathrm{GeV}$ by using the available experimental and tabulated values in that energy range. There are some studies of validation of few electromagnetic physics models only up to $1.2 \mathrm{GeV}$ [8]. So there is a need to choose and validate other Geant4 
electromagnetic physics models suitable up to $10 \mathrm{GeV}$. So it has been tested both Geant 4 electromagnetic and hadronic physics models suitable for space radiation interaction and transport in that whole energy range. For the simulation purpose a Dell laptop with configuration- 8 GB RAM, i5 processor, $2.20 \mathrm{GHz}$ clock speed and windows 10 Operating System is used in the present work. The Geant4 toolkit Version 4.10.1. p02 software is installed on this laptop to do the simulation work.

\section{Physics Models}

The electromagnetic and hadronic interactions are simulated separately by using the Physics Models of Geant4 Version 4.10.1. p02. G4DecayPhysics is also used as general physics model in both cases in addition to the respective physics models selected.

G4EmStandardPhysics_option3 is used for the simulation of electromagnetic interactions. By choosing this physics the following electromagnetic processes are automatically applied for different particles in different energy ranges: (only few important particles and processes are shown)

Table 1. Physical processes applicable for different particles as part of G4EmStandardPhysics_option3.

\begin{tabular}{|c|c|c|c|}
\hline Particle & Physical Process & Physics Model & Energy Range \\
\hline \multirow[t]{4}{*}{ proton } & multiple scattering & $\begin{array}{l}\text { Urban multiple } \\
\text { scattering model }\end{array}$ & $100 \mathrm{eV}-10 \mathrm{TeV}$ \\
\hline & hadron ionization & Bragg model & $0 \mathrm{meV}-2 \mathrm{MeV}$ \\
\hline & & Bethe-Bloch model & $2 \mathrm{MeV}-10 \mathrm{MeV}$ \\
\hline & $\begin{array}{l}\text { hadron } \\
\text { bremsstrahlung }\end{array}$ & & $0 \mathrm{meV}-10 \mathrm{TeV}$ \\
\hline \multirow[t]{3}{*}{$\begin{array}{l}\text { alpha } \\
\text { particle }\end{array}$} & multiple scattering & $\begin{array}{l}\text { Urban multiple } \\
\text { scattering model }\end{array}$ & $100 \mathrm{eV}-10 \mathrm{TeV}$ \\
\hline & ion ionization & Bragg ion model & $0 \mathrm{meV}-7.9452 \mathrm{MeV}$ \\
\hline & & Bethe-Bloch model & 7.9452 MeV-10TeV \\
\hline \multirow[t]{2}{*}{ ions } & multiple scattering & $\begin{array}{l}\text { Urban multiple } \\
\text { scattering model }\end{array}$ & $0 \mathrm{meV}-10 \mathrm{TeV}$ \\
\hline & ion ionization & Parametric ICRU73 & $0 \mathrm{meV}-10 \mathrm{TeV}$ \\
\hline
\end{tabular}

Hadronic interactions are simulated by using a suitable choice of three physics models: G4HadronElasticPhysics for hadron elastic processes, G4HaronPhysics QGSP_BERT_HP for hadron inelastic processes and G4IonQMDPhysics for generic ions.

By choosing this Physics the following hadronic processes are automatically applied for different particles in different energy ranges: (only few important particles and processes are shown)

Table 2. Physical processes applicable for different particles as a part of G4HaronPhysicsQGSP_BERT_HP and G4IonQMDPhysics.

\begin{tabular}{llll}
\hline Particle & Physical Process & Physical Model & Energy Range \\
\hline proton & proton inelastic & QGSP model & $12 \mathrm{GeV}-100 \mathrm{TeV}$ \\
& & $\begin{array}{l}\text { FTFP model } \\
\text { Bertini cascade } \\
\text { model }\end{array}$ & $9.5 \mathrm{GeV}-25 \mathrm{GeV}$ \\
& & meV-9.9 GeV \\
alpha & \multirow{2}{*}{$\begin{array}{l}\text { Binary light ion } \\
\text { particle /ion }\end{array}$} & cascade model & $0 \mathrm{meV}-110 \mathrm{MeV}$ \\
& & QMD model & $100 \mathrm{MeV}-10 \mathrm{GeV}$ \\
& & FTFP model & $9.9 \mathrm{GeV}-1 \mathrm{TeV}$ \\
\hline
\end{tabular}

\section{Methods}

For the validation of Geant4 physics models it has been considered four parameters: energy loss, electronic stopping power, isotopic production cross-section and total fragmentation cross-section.

Energy-loss of charged particle is given by:

$$
\text { Energy- loss }=-\frac{d E}{d x}(\text { in } \mathrm{MeV} / \mathrm{cm})
$$

where $\mathrm{dE}$ is the average energy imparted by the particle (in $\mathrm{MeV}$ )

and $\mathrm{dx}$ is the distance travelled by the particle (in $\mathrm{cm}$ ).

In simulations $\mathrm{dx}$ is considered as the thickness of target material and is chosen to be small so that it is always smaller than the range of incident particle in a given target material.

The stopping power is given by:

Stopping power $=$ energy loss/density of target material (2)

(in $\mathrm{MeV} \mathrm{cm} / \mathrm{g}$ )

The total proton-proton production cross-sections are calculated by:

Isotope production cross-section

$$
(\sigma)=\frac{\mathrm{N}_{\text {iso }}}{\mathrm{N}_{\mathrm{t}} \times \mathrm{xN}_{\text {in }}}(\text { in } \mathrm{mb})
$$

where $\mathrm{N}_{\text {iso }}$ is the number of isotopic species produced

$N_{t}$ is the number of target atoms per unit volume (in $/ \mathrm{cm}^{3}$ )

$\mathrm{t}$ is the thickness of the target (in $\mathrm{cm}$ )

and $N_{\text {in }}$ is the number of incident particles

The total fragmentation cross-sections for Fe 26 ions are calculated by:

Total fragmentation cross-section

$$
\left(\sigma_{\text {tot }}\right)=\frac{A_{T} \ln \left(N_{\text {in }} / N_{\text {out }}\right)}{\rho t N_{A v}}(\text { in mb) }
$$

where $A_{T}$ is the mass number of the target material $N_{\text {in }}$ is the number of incident ions before the target

$N_{\text {out }}$ is the number of incident ions after the target

$\rho$ is the density of the target material (in $\mathrm{g} / \mathrm{cm}^{3}$ )

$\mathrm{t}$ is the thickness of the target (in $\mathrm{cm}$ )

and $N_{A v}$ is the Avagadro Number

In carrying out the simulations - the number of particles, intensity and focusing of beam are considered as factors which play no role in the evaluation of isotopic and total fragmentation cross sections. The number of particles used in the simulation only affects the amount of statistical error which is of order $\frac{1}{\sqrt{N}}$, where $\mathrm{N}$ is the number of particles. So in the simulations the sufficient numbers of particles are considered to get the reliable results. In addition to this the moderate thickness of target materials are necessary to produce the sufficient number of fragments of an isotope. The target materials in the simulation are selected on the basis of their current use and future prospects for making of space vehicles. The particles used in the simulation are the important components of space radiation. The energies of those particles are considered in the energy range of nuclear component of cosmic radiation arriving at the earth's orbit. 


\section{Results}

It has been simulated few experiments by using Geant4 toolkit and simulated parameters are compared to the experimental and tabulated values in the present work. For electromagnetic interactions energy-loss and electronic stopping powers are calculated whereas for hadronic interactions isotope production cross-sections and total fragmentation cross-sections are calculated in the simulations.

\subsection{Electromagnetic Physics Simulation Results}

Aluminum slab of area $10 \mathrm{mx} 10 \mathrm{~m}$ and thickness $20 \mathrm{~g} / \mathrm{cm}^{2}$ is constructed and a beam of 10000 protons emerging from a plane of same area as slab made to incident normally on the slab. The energies of protons are chosen from $200 \mathrm{MeV}$ to $10000 \mathrm{MeV}$ and the corresponding stopping powers are calculated. The values calculated in the simulation are compared with the NIST database values [5]. The values are in good agreement with the NIST data for proton energies near $10000 \mathrm{MeV}$ as reported in Figure 1.

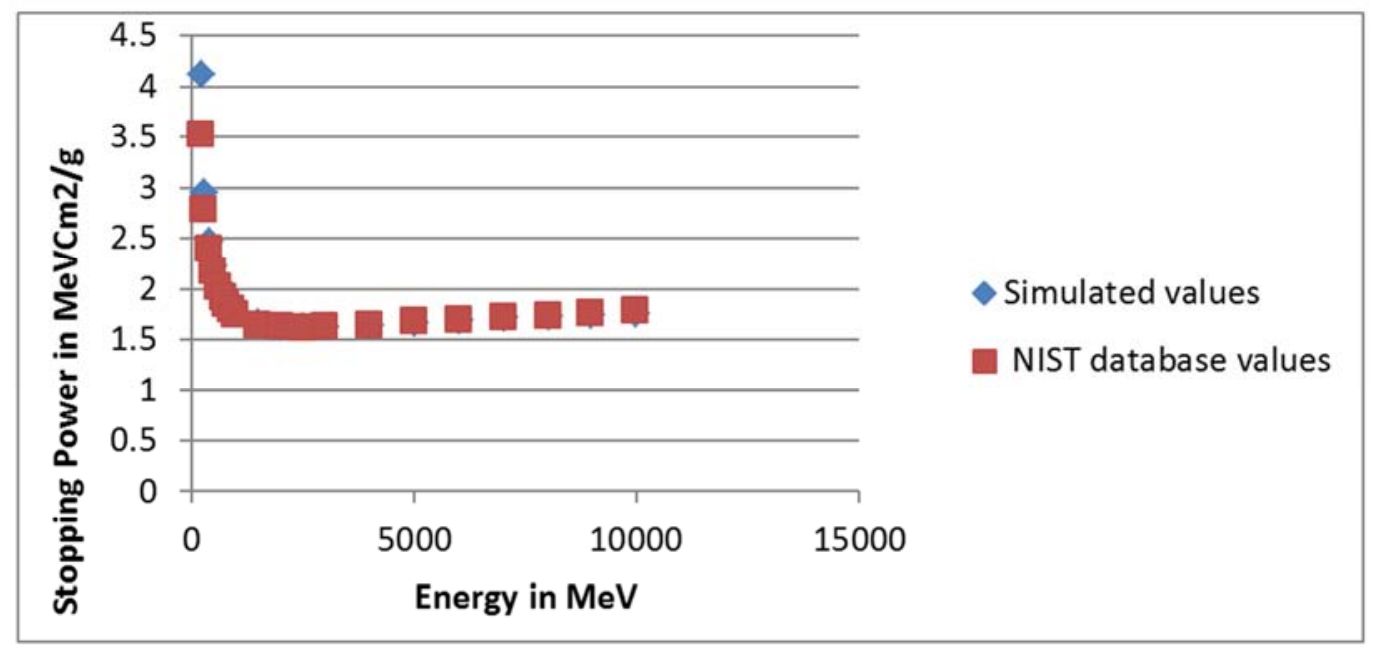

Figure 1. Comparison of simulated and tabulated stopping power values of protons in Aluminum.

It has been found the residual energy of $40 \mathrm{MeV}$ alpha particles in the aluminum slab of area $10 \mathrm{mx} 10 \mathrm{~m}$ and thickness varying from $15.2 \mathrm{mg} / \mathrm{cm}^{2}$ to $155.2 \mathrm{mg} / \mathrm{cm}^{2}$. The simulated values are in good agreement with the experimental values [13] when the thickness of aluminum slab is small as reported in Figure 2.

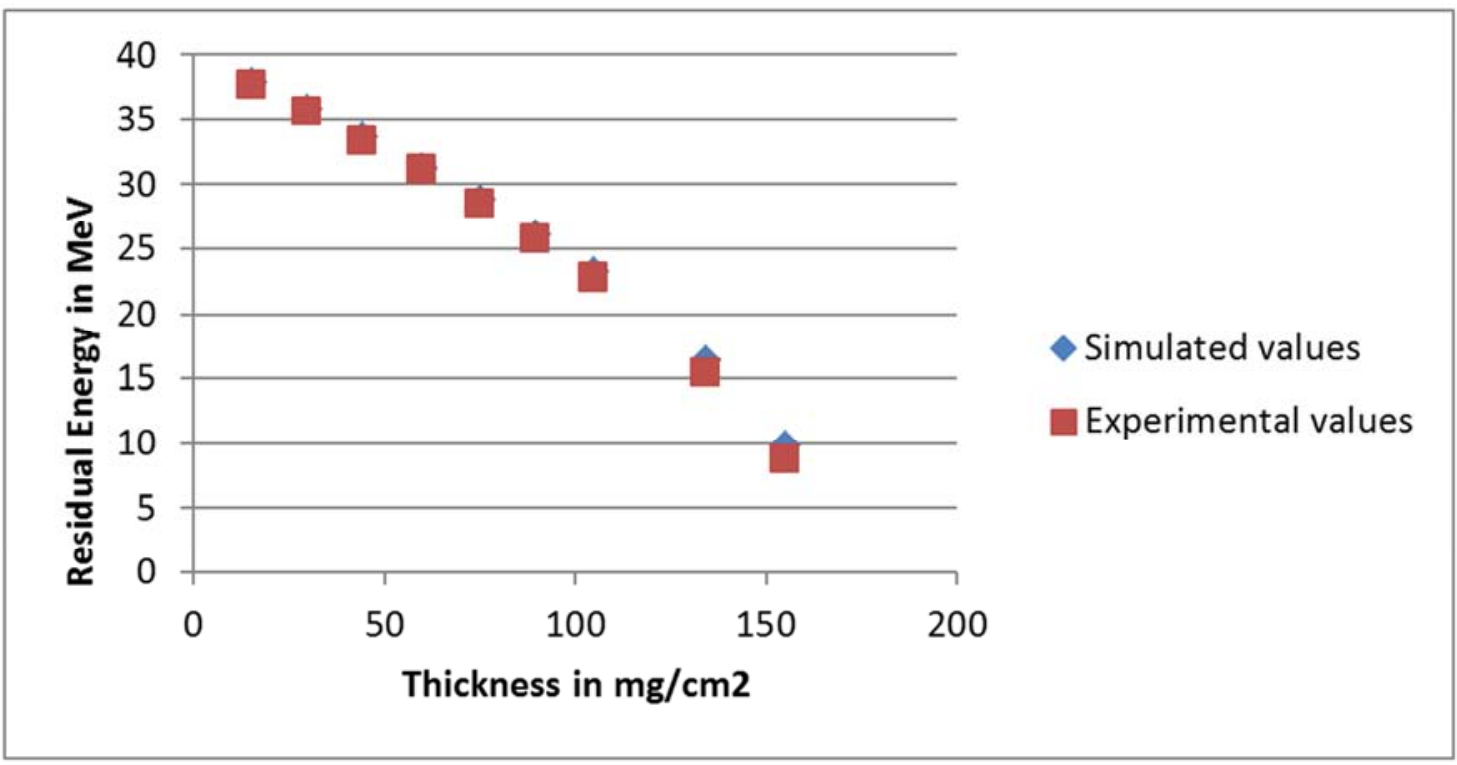

Figure 2. Comparison of the simulated and experimental values of residual energies of alpha particles in different thicknesses of Al material.

It has also been found the energy-loss of alpha particles in detector material silicon of area $10 \mathrm{mx} 10 \mathrm{~m}$ and thickness $0.48 \mathrm{~g} / \mathrm{cm}^{2}$. Alpha particles emerging from plane of same area as Si slab allowed to incident normally on Si slab with energies ranging from 175 to $910 \mathrm{MeV}$. The energy-loss values are simulated and compared with experimental values [13]. The values are in good agreement at higher energies as reported in Figure 3. 


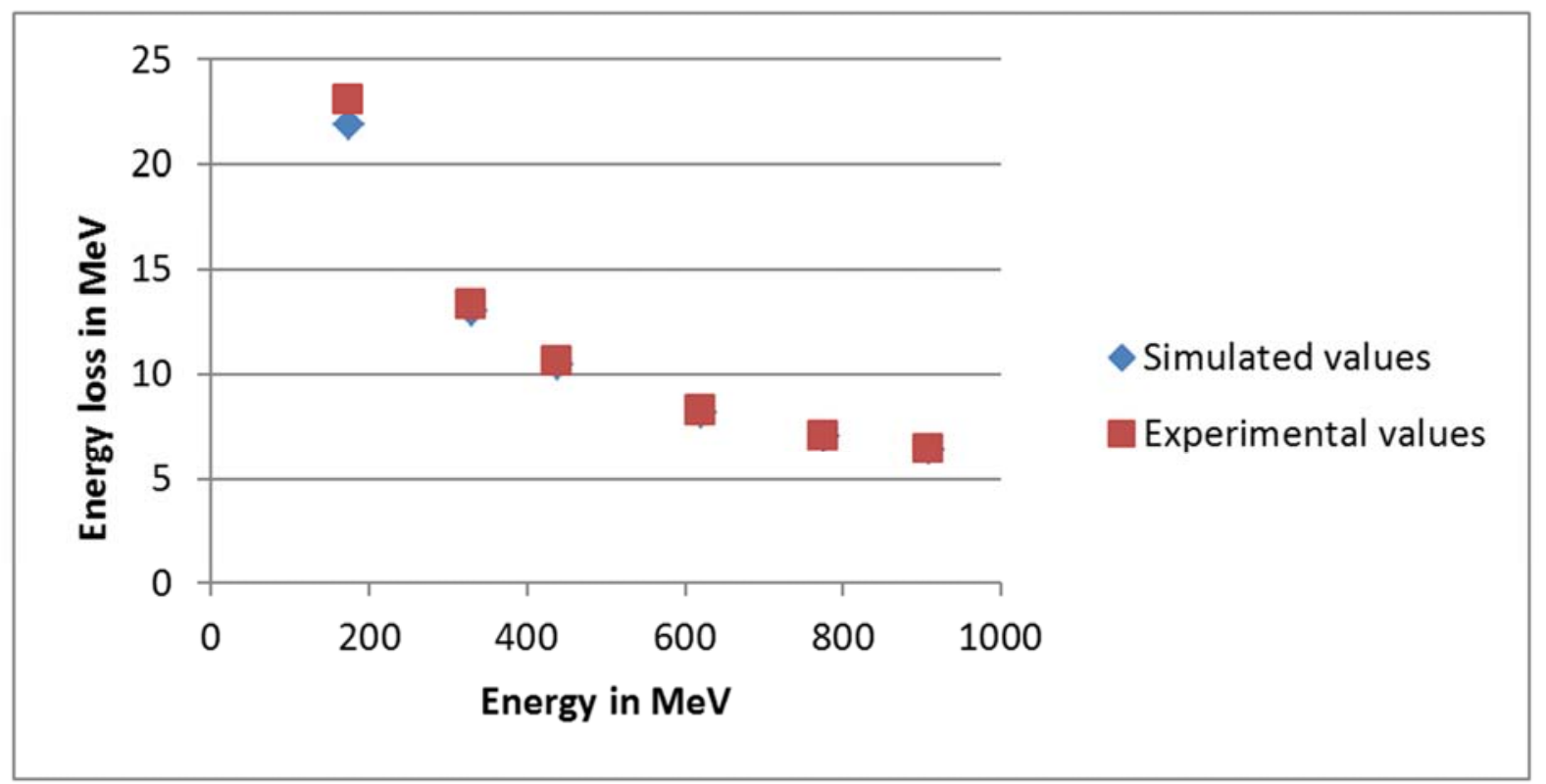

Figure 3. Comparison of simulated and experimental energy-loss values at different energies of alpha particles in Silicon slab.

Stopping power values of alpha particles in aluminum slab of area $10 \mathrm{mx} 10 \mathrm{~m}$ and thickness of $5 \mathrm{~g} / \mathrm{cm}^{2}$ are simulated. For this purpose $\mathrm{Al}$ slab is made to incident normally by 10000 alpha particles emerging from a plane of same area as aluminum slab and simulated values are compared with the
NIST database vales. The values are in good agreement with the NIST database values as in the case of protons. The values are approaching the NIST data base values when energy increases from $600 \mathrm{MeV}$ to $1000 \mathrm{MeV}$ as reported in Figure 4.

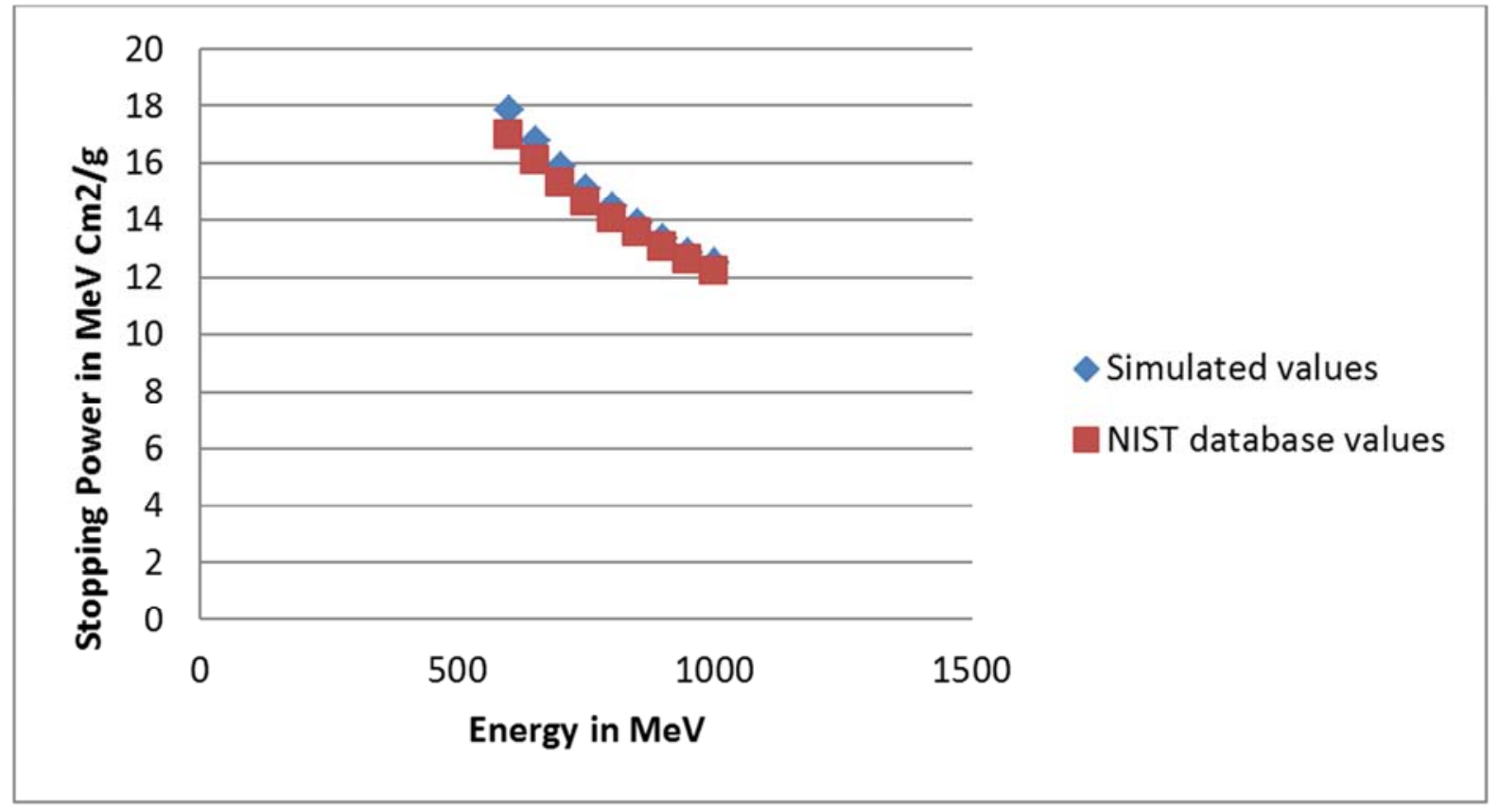

Figure 4. Comparison of simulated and tabulated stopping power values of alpha particles in aluminum slab.

Stopping power values of Fe ions in aluminum slab of area $10 \mathrm{mx} 10 \mathrm{~m}$ and thickness of $10 \mathrm{mg} / \mathrm{cm}^{2}$ are also simulated and compared to the tabulated values [10]. For this purpose Al slab is made to incident normally by $10000 \mathrm{Fe}$ ions emerging from a plane of same area as aluminum slab. The stopping-power values for Fe ions in $\mathrm{Al}$ are in good agreement with tabulated values from 300 to $500 \mathrm{MeV}$ /nucleon as reported in Figure 5. 


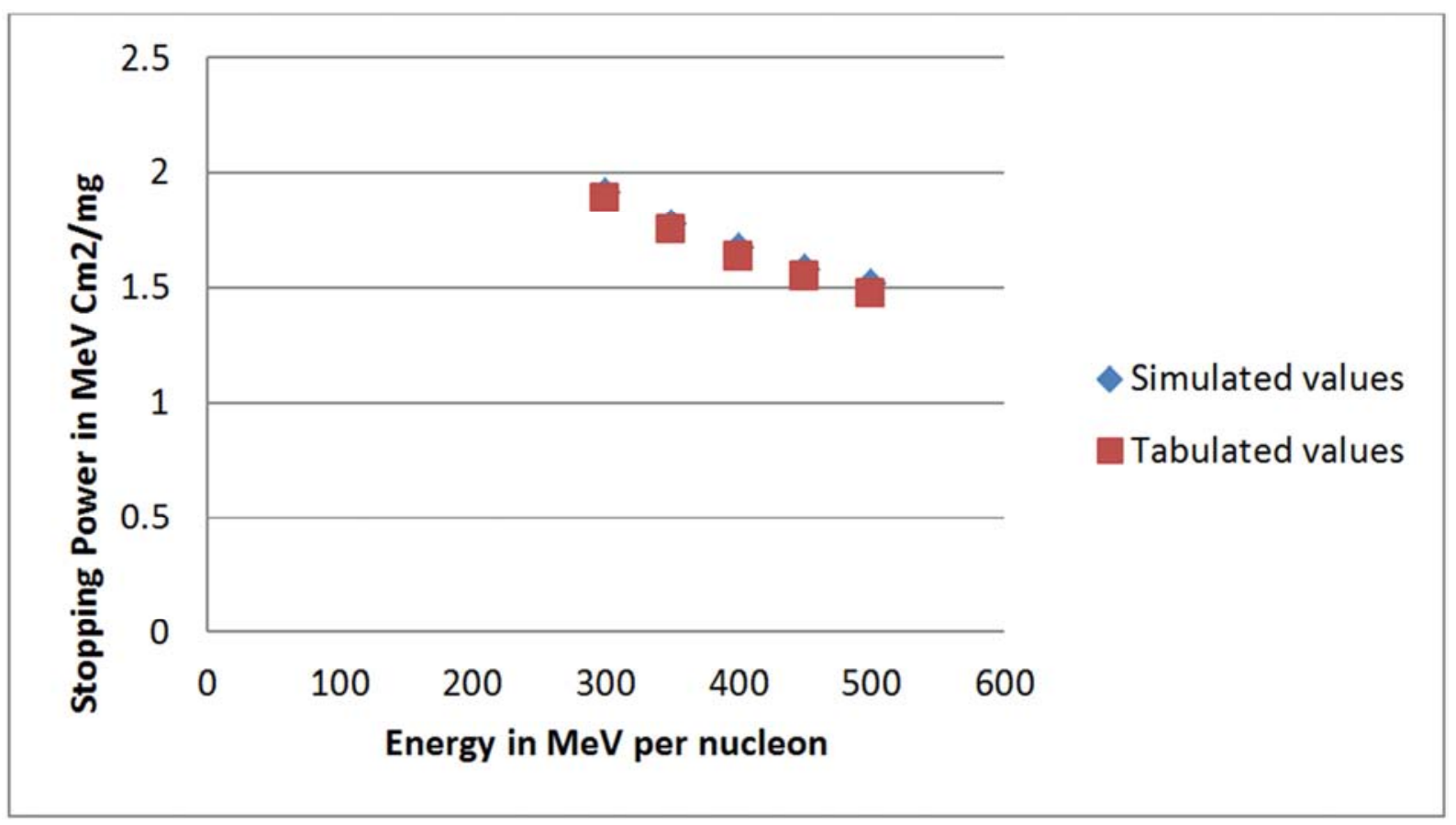

Figure 5. Comparison of simulated and tabulated stopping-power values of Fe in Al.

\subsection{Hadronic Physics Simulation Results}

In order to test hadronic physics an experiment [14] is constructed and total proton-proton cross-sections for materials hydrogen and polyethylene are calculated. First a liquid hydrogen cylinder of $16 \mathrm{~cm}$ diameter and $75 \mathrm{~cm}$ thickness is constructed and a beam of 10000 protons with different energies made to incident parallel to the length of the cylinder. The simulated proton cross-section values for liquid hydrogen are in good agreement with the experimental values from $179 \mathrm{MeV}$ to $555 \mathrm{MeV}$ as reported in Figure 6.

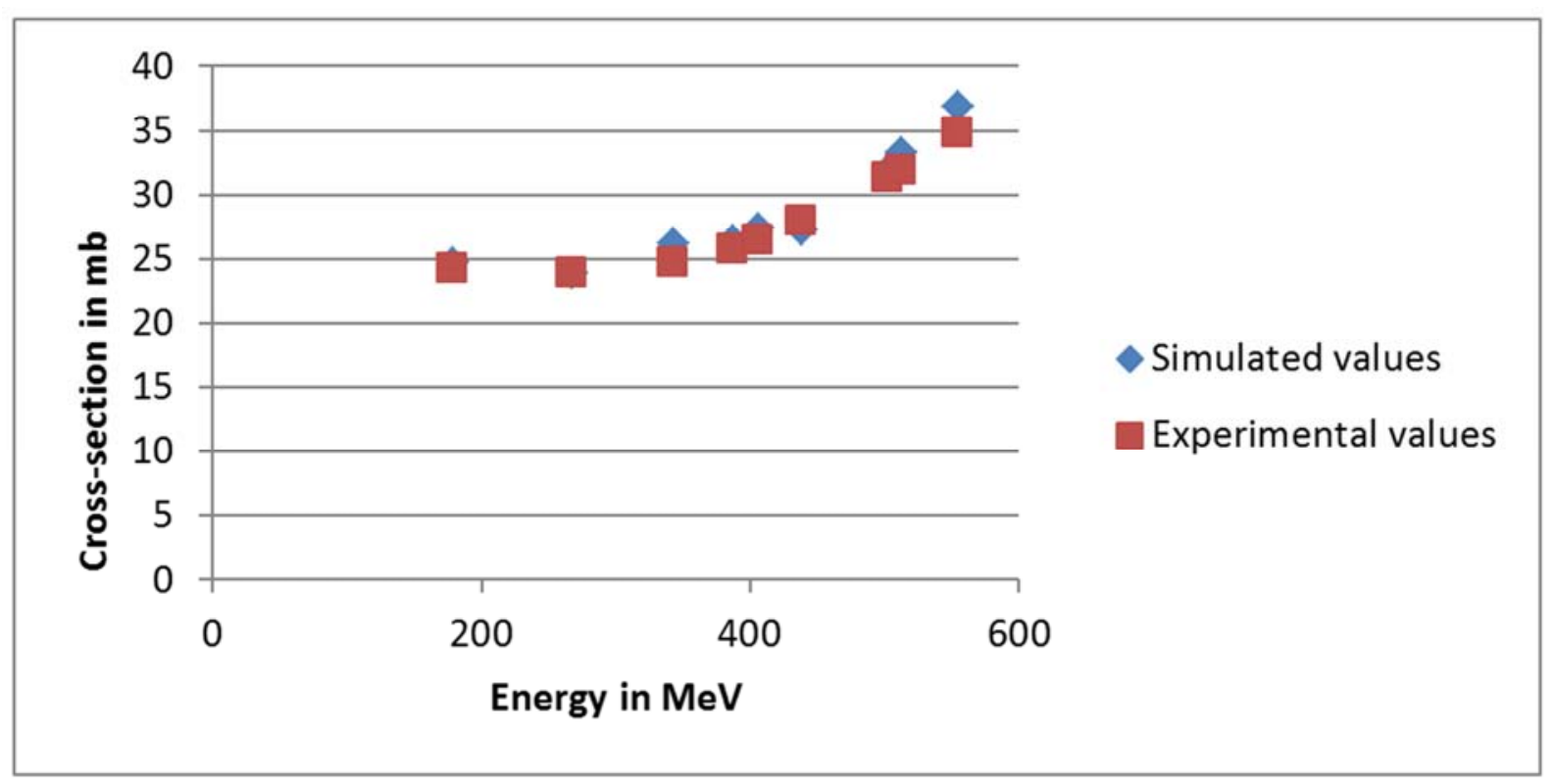

Figure 6. Comparison of proton-proton production cross-sections at different energies of protons in liquid hydrogen material.

The similar experiment [14] with a cylinder of polyethylene of $16 \mathrm{~cm}$ diameter and $5 \mathrm{~cm}$ of thickness is constructed and a beam of 10000 protons with different energies are allowed to incident parallel to the axis of the cylinder. The simulated proton cross-section values for polyethylene target are also in agreement with the experimental values from energies $463 \mathrm{MeV}$ to 550 $\mathrm{MeV}$ as reported in Figure 7. 


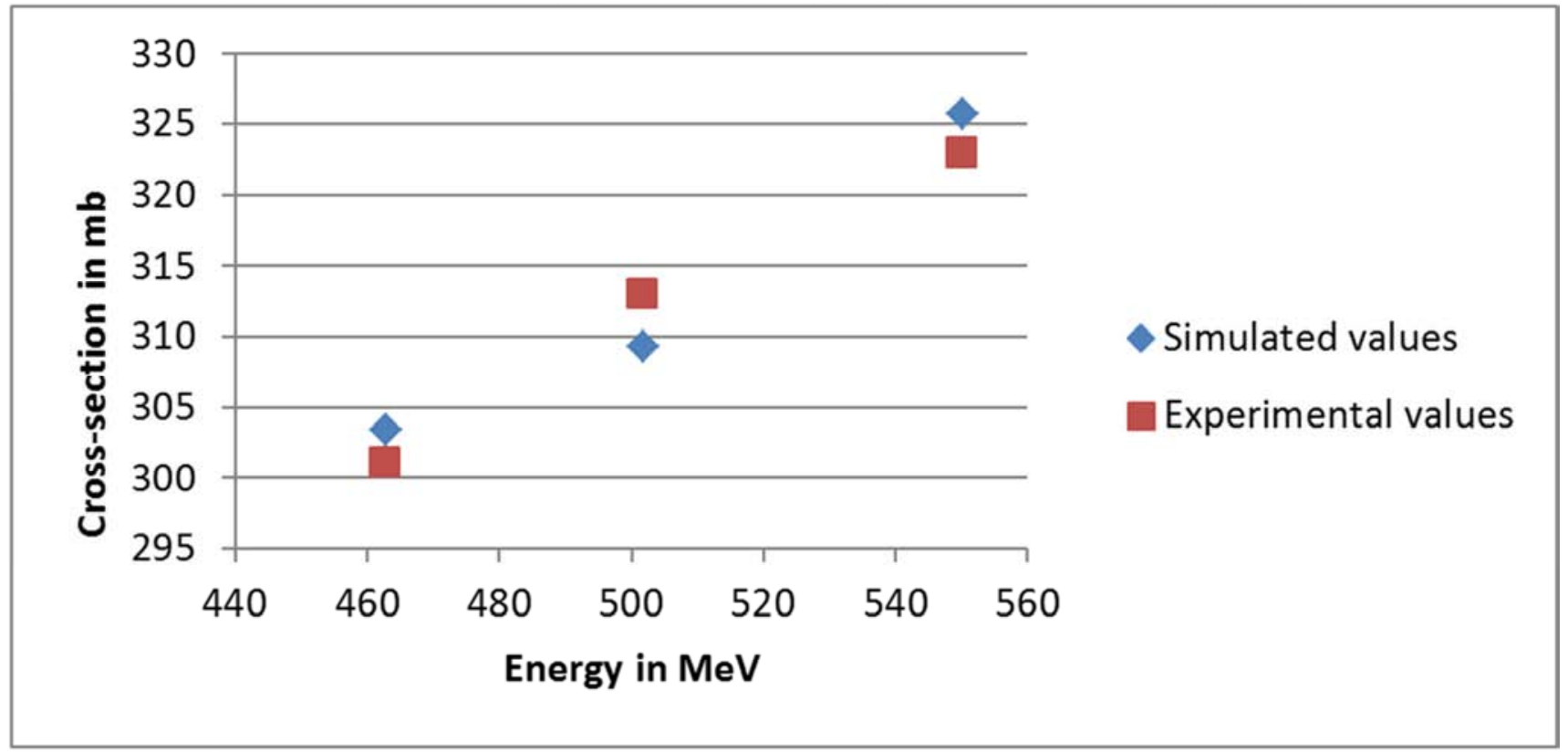

Figure 7. Comparison of proton-proton cross-sections at different energies of protons in polyethylene material.

It has also been simulated an experiment [6] and calculated total fragmentation cross-sections for $\mathrm{Fe}$ ions in $\mathrm{Al}$ and polyethylene targets in the energy range of $0.3 \mathrm{~A} \mathrm{GeV}$ to 10 $\mathrm{A} \mathrm{GeV}$. For this purpose a polyethylene slab of area $5 \mathrm{~m} \times 5$ $\mathrm{m}$ and thickness $1 \mathrm{~cm}$ is used as target and a beam of $2000 \mathrm{Fe}$ ions is made to incident normally on the target. The calculated total fragmentation cross-section values are in agreement with the experimental values within the experimental error of $10 \%$, except at lower energies as reported in Figure 8.

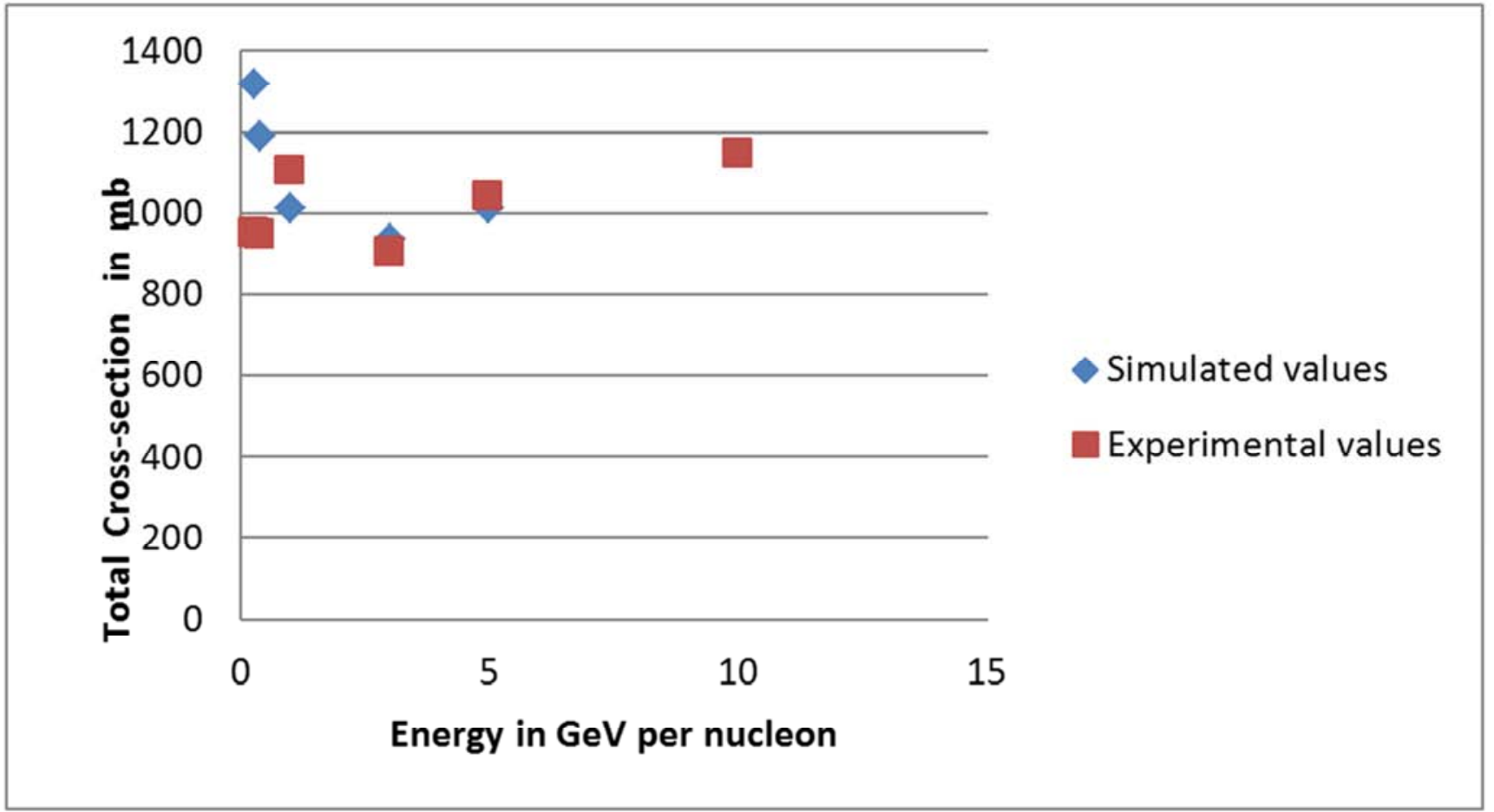

Figure 8. Comparison of total fragmentation cross-sections at different energies of Fe ions in polyethylene material.

It has also been simulated the total cross-section values of $\mathrm{Fe}$ ions in $\mathrm{Al}$ material and compared the values with the experimental values [6]. For this purpose an aluminum slab of $5 \mathrm{~m} \mathrm{x} 5 \mathrm{~m}$ area and $1 \mathrm{~cm}$ thickness is used as target and a beam $2000 \mathrm{Fe}$ ions is made to incident normally on the target. The calculated cross-section values are within the experimental error limit of about $10 \%$, except at low energies as reported in Figure 9. 


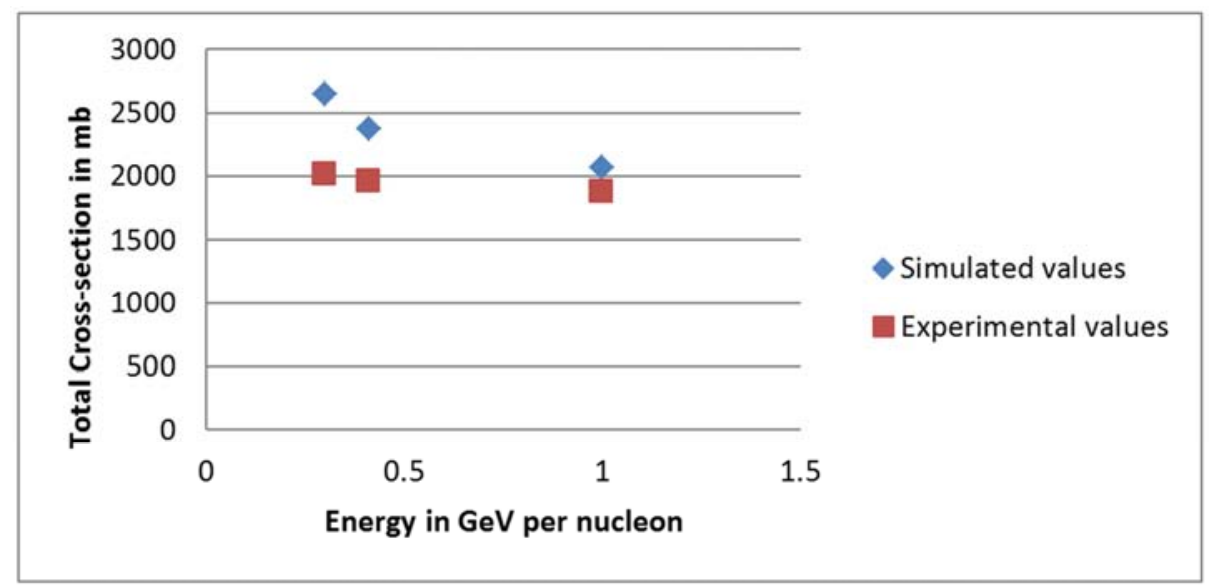

Figure 9. Comparison of total fragmentation cross-sections at different energies of Fe ions in aluminum material.

\section{Discussion}

The electromagnetic models used in the simulations are well describing the interactions for protons. The simulation results for electronic stopping powers are well agreeing with NIST database values particularly at high energies. In case alpha particles simulated values of energy-loss for Si material are in good agreement with experimental values near 910 $\mathrm{MeV}$. In case of alpha particles of $40 \mathrm{MeV}$ on the aluminum material of various thicknesses the simulated values of residual energy are also in good agreement with experimental values at the lower thickness values of the material. Furthermore simulated values of stopping powers for alpha particles in Al material are also in good agreement with NIST database values as one goes from $600 \mathrm{MeV}-1000 \mathrm{MeV}$. The physical model applied in simulations in this energy range is Bethe-Bloch model with Shell, Barkas, and Bloch and density effect corrections. NIST data tables are also developed based on the same theory in this energy range. So any small discrepancies in results are due to the simulation setup but not due to the physical models. This is true because it is observed in the simulations that by reducing the Al target thickness for alpha particles of given energy the discrepancies are largely reduced.

Simulated values of stopping powers for heavy ion $\mathrm{Fe}$ in $\mathrm{Al}$ material are also agreeing with the tabulated values from 300 $\mathrm{MeV}$ to $500 \mathrm{MeV}$ [10]. In Geant4 simulations parameterized ICRU73 model is used whereas in the tabulated values a new parameterized model is used. But still the results are in good agreement with each other. So in selected energy range of simulation both models are giving equivalent description of electromagnetic interactions of heavy ions like Fe. But the experimental values and their agreement with these values only confirm the validity of each model.

So at high energies and small thickness of materials electromagnetic models are fairly well describing the electromagnetic interactions. The reason for this is, for the small thickness at a given energy or for the given thickness at a relatively high energy matches the conditions of applicability of the energy loss or stopping power formula used in the simulations. So any small discrepancies at lower energies need not be taken as the flaw in the physical models of the interactions. Hence the models used for the simulation of electromagnetic interactions of space radiation can be used for space radiation protection studies.

The hadronic physics models are very well describing the proton-proton collisions and subsequent proton production cross-sections from $179 \mathrm{MeV}$ to $555 \mathrm{MeV}$. The proton proton production cross-section values are within the limits of experimental errors. The physical model used here for protons is Bertini Cascade Model. Even though this model giving good results for protons, it does not work for ions. The simulated total fragmentation cross-section values for Fe ions in polyethylene and aluminum material from $0.30 \mathrm{GeV}$ to 10 $\mathrm{GeV}$ are agreeing with experimental values except at low energies. At low energies the values of cross-sections are beyond the experimental errors of about $10 \%$. The physics model used in Geant4 simulations here is the Ion QMD model. Since this is the naïve QMD model which can be applied directly in Geant4, it is not fully describing the ion interactions. This model is giving discrepancies beyond the limits of experimental error at low energies in case of both polyethylene and aluminum. So an improved QMD model can be used to get the more precise results. However, this model can be used for the preliminary analysis of space radiation protection because the results are good at the peak of GCR spectrum which is around $1 \mathrm{GeV} /$ nucleon for all ions. So the hadronic interaction models used in present simulations can be used for an approximate analysis of space radiation protection.

In the present simulation work it has been used the Geant 4 Reference Physics Lists. Instead of this one can use - for each particle and for each process a specific physics model of Geant 4 or its interfaces to other models from other codes. So there is a scope for further optimization of the physics models used in the space radiation protection studies based on the validation results of the present models used in this simulation work.

\section{Conclusions}

The selected physics models for both electromagnetic as 
well as hadronic processes of components of space radiation - protons, alpha particles as well as Fe ions are tested and validated. Validation tests indicate that electromagnetic physics models for space radiation protons, alpha particles and $\mathrm{Fe}$ ions are agreeing well with the tabulated values. Furthermore, the experimental values of energy loss and residual energy of alpha particles are also in good agreement with simulation results at the small thicknesses target materials or at high energies of alpha particles. Since small discrepancies at large thicknesses or low energies are only the simulation setup issue, the electromagnetic models are well describing the space radiation interactions. The protonproton production cross-section values are also agreeing well with the experimental results within the given error limit of $1-3 \%$ in the tested energy range. The total fragmentation cross-sections for $\mathrm{Fe}$ ions are also agreeing with the experimental values within the experimental error of about $10 \%$, except at low energies. Furthermore results at the peak of GCR spectrum which is around $1 \mathrm{GeV} /$ nucleon are agreeing well with the experimental values for both polyethylene and aluminum. So hadron physics models are approximately well describing the space radiation interactions. So the electromagnetic and hadronic physics models used in this simulation work can be used for the space radiation protection studies. Furthermore, based on the results of this work, there is the scope for further optimization of physics used in space radiation protection studies.

\section{Acknowledgements}

I wish to thank Prof S. R. Hassan, Institute of Mathematical Sciences (I. M. Sc) Chennai, India, for reading the manuscript of the paper and giving valuable suggestions. I also wish to thank the Geant4 collaboration for utilizing their Geant4 toolkit software version 4.10.1.p02 in the simulations.

This work is supported by University Grants Commission (U. G. C) of India under Minor Research Project Grant No. F MRP- 4791/14 (SERO/UGC).

\section{References}

[1] Agostinelli, S., Allison, J., Amako, K., et al., 2003, 'GEANT4- a simulation toolkit', Nucl. Instr. and Meth. A, vol.506, pp. $250-303$.

[2] Allison, J., Amako, K., Apostolakis, J., et al., 2006, 'Geant4 developments and applications', IEEE Trans. Nucl. Sci., vol.53, no.1, pp. 270-278.

[3] Apostolakis, J., Asai, M., Bogdanov, A. G., et al., 2009, 'Geometry and physics of the Geant4 toolkit for high and medium energy applications', Radiation Physics and Chemistry, vol. 78, pp. 859-873.

[4] Apostolakis, J., Asai, M., Bagulya, A., et al., 2015, 'Progress in Geant4 Electromagnetic Physics Modelling and Validation', Journal of Physics: Conference Series, 664072021. doi: 10.1088 / 1742-6596/ 664/7/072021

[5] Berger, M. J., Coursey, J. S., Jucker, M. A., et al., 2005, 'ESTAR, PSTAR and ASTAR: Computer Programs for Calculating Stopping Power and Range Tables for Electrons, Protons and Helium Ions (version 1.2.3)' http://www. nist. gov/Star> [29 Dec 2015]

[6] Cecchini, S., Chiarusi T., Giacomelli, G., et al., 2008, 'Fragmentation cross sections of $\mathrm{Fe}^{26+}, \mathrm{Si}^{14+}$ and $\mathrm{C}^{6+}$ ions of 0.3-10A GeV on polyethylene, CR39 and aluminum targets', Nuclear Physics A, vol.807, pp. 206-213.

[7] Durante, M., and Francis A. Cucinotta., 2011, 'Physical basis of radiation protection in space travel', Rev. Mod. Phys., vol.83, no.4, pp. 1245-1281.

[8] Filomena Loffredo, Mariagabriella Pugliese, Maria Quarto et al., 2015,'Validation of Electromagnetic Physical Processes with Software SPENVIS',Vol.4,no.6,pp.275-278. doi: 10.11648 / j. ajep.20150406.12

[9] Geant4 Physics Reference Manual 〈www://geant4.web.cern.Ch /geant4/UserDocumentation/UserGuides/PhysicsReferenceMa nual/fo/PhysicsReferenceManual. pdf〉 [14 Oct 2015]

[10] Hubert, F., Rimbot, R., Gauvin, H., et al., 1990, 'Range and Stopping-Power Tables for $2.5-500 \mathrm{MeV} /$ nucleon Heavy Ions in Solids', Atomic Data and Nuclear Data Tables, vol.46, pp. 1-213.

[11] Li-Chun Wang, Dong-Hai Zhang, Shiwei Yan et al., 2012, 'Fragmentation cross sections of $56_{\mathrm{Fe}}$ at $471 \mathrm{~A} \mathrm{MeV}$ on Al, C and CH2 Targets', Acta Physica Polonica B, vol.43, no.8, pp. 1769-1782.

[12] Makato Asai, Andrea Dotti., Marc Verderi., et al., 2014, 'Recent Developments in Geant4', Ann. Nucl. Energy. 〈http://dx. doi.org /10.1016/j. anucene.2014.08.021〉

[13] Mudundi R. Raju, 1965, 'Heavy particle studies with silicon detectors', University of California Research Laboratory Report, UCRL- 16354.

[14] Schwaller, P., Pepin, H., Favier, B., et al., 1979, 'Proton total cross-sections on $1_{H}, 2_{H}, 4_{H e}, 9_{B e}, \mathrm{C}$ and $\mathrm{O}$ in the energy range 180 to $560 \mathrm{MeV}$, Nucl. Phy. A, vol.316, pp. 317-344. 